\title{
A New Combination of Local Appearance Based Methods for Face Recognition under Varying Lighting Conditions
}

\author{
Heydi Méndez-Vázquez, Edel García-Reyes, and Yadira Condes-Molleda \\ Pattern Recognition Department, Advanced Technologies Application Center \\ 7th Avenue \# 21812, Siboney, Playa, Cuba, 12200 \\ $\{$ hmendez, egarcia, ycondes $\}$ @ cenatav.co.cu
}

\begin{abstract}
This paper presents a different way of using DCT and LBP to compensate for illumination variations in face recognition with only one sample image per person. The sensitiveness to lighting variations of the LBP and DCT methods was investigated and from it emerged the proposed new method, consisting in discarding low-frequency DCT coefficients in the logarithm domain in a local way as a preprocessing step, and applying LBP method to represent the facial features. Experimental results on the Yale B database show that the proposal improves the performance of the original methods and their existing extensions.
\end{abstract}

Keywords: local appearance based methods, face recognition, LBP, DCT, illumination invariants.

\section{Introduction}

Automatic face recognition has been widely used in a variety of applications in the last years [1]. Although there are a number of face recognition algorithms which work well in constrained environments, face recognition is still an open and very challenging problem in real outdoor applications.

Variations in lighting are among the most affecting performance of face recognition systems [2]. To attack the problem of face recognition under illumination variation, several algorithms have been proposed. Nevertheless, their performances are still far from ideal and many of them require a large number of training images.

Face recognition methods based in one single image per person can be classified into two categories taking into account the type of features that they use: geometric based methods and appearance based methods.

Appearance based methods, have been the dominant techniques in the last years. Such an approach generally operates directly on the pixel intensities or other imagebased representation and has greatly improved the effectiveness and efficiency of face recognition systems [3]. These kinds of methods can be used either in a holistic or in a local way. The holistic methods identify a face using as input a vector that represents the whole face image. The local ones use the information of the face image localities for the recognition purpose. In [4] it is shown that local normalization methods are more invariant to illumination variations than global ones. Then, a variety of local 
appearance based methods for face recognition has been developed. Among them, the Local Binary Patterns (LBP) [5] and the local Discrete Cosine Transform (DCT) [6], show the best results in front of lighting variations.

The LBP operator is by definition invariant to monotonic gray variations. In a recent comparison between the different uses of the LBP [7], the best performance was shown by a method [8] that uses this operator as a preprocessing step.

The DCT method is also claimed to be less sensitive to illumination variations. When using DCT, usually the feature vector used for classification is formed with the low - frequency coefficients because they contain most of the face image information. Though, in [9] a different way of using DCT to compensate for illumination variations is presented, discarding low - frequency DCT coefficients in the logarithm domain, presenting promising results.

The aims of this paper is to prove the following hypothesis: (1) The LBP operator is sensitive to large variations in illumination conditions; (2) The DCT method outperforms the LBP facing the illumination problems; (3) The use of local DCT in the logarithm domain as preprocessing and LBP to represent the facial structures outperforms the results of the other approaches in which these methods are used.

This paper is organized as follows. Section 2 analyzes the LBP and DCT methods as illumination invariants. Section 3 presents the proposed method. Section 4 reports on the experimental results. Finally, Section 5 concludes the paper.

\section{Local Appearance Based Methods as Illumination Invariants}

Local appearance based methods generally involve four steps: local region partition, feature extraction, feature selection and classification. For the problem of image recognition under varying illumination conditions, the first two stages are the fundamental ones, and many different methods have been proposed [3]. If the reports of the existing methods are analyzed, it can be noticed that the extraction of illumination invariant local features are not a solved problem up to now, being LBP and DCT methods the ones that show the best results. For that reason we decided to study both of them in more details.

\subsection{Local Binary Patterns as Illumination Invariant}

The use of this operator in face recognition was introduced in [5] and different extensions from the original operator have appeared afterwards [7]. The original LBP operator labels the pixels of an image by thresholding the $3 \times 3$ - neighbourhood of each pixel with the centre value and considering the result as a binary number called the LBP code. Then the image is divided into rectangular regions and histograms of LBP codes are calculated over each of them. Finally, the histograms of each region are concatenated into a single one that represents the face image and a dissimilarity measure is used to compare the histograms of different images.

The idea behind using the LBP features is that face images can be seen as a composition of micro-patterns such as edges, spots and flat areas, as can be apreciated in Fig. 1. With this operator, these patterns can be represented only taking into account if the surrounding pixel values are bigger or smaller than the center pixel value. Many 


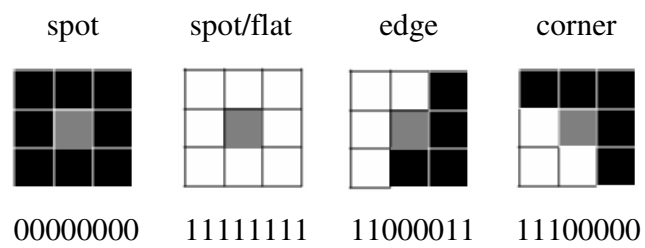

Fig. 1. Examples of micro-patterns represented by LBP operator

papers declare that the LBP operator is robust to illumination variations [8] [10]; this means that the operator is able to describe the facial features independently from the variations of illumination that may be affecting the image.

If we look at the pixels under the Lambertian model, we can consider:

$$
I_{c}=\rho_{c} \cdot n_{c}{ }^{T} \cdot s_{c} \quad \text { and } \quad I_{1}=\rho_{1} \cdot n_{1}{ }^{T} \cdot s_{1}
$$

where $\rho$ is the albedo, $n^{T}$ the normals to the object surface and $s$ the light source falling on, $I_{C}$ be the center pixel intensity and $I_{I}$ the pixel intensity of a neighbour of $I_{C}$. Both $\rho$ and $n^{T}$ depend on the physical properties and the shape of the surface respectively, and they are expected to be similar in a little spatial neighbourhood, except in those in which important changes on the surface occurs. So, in most cases, the difference between both pixels intensities described by the LBP operator really represents the changes in the incident illuminations:

$$
I_{1}-I_{c}=\rho \cdot n^{T} \cdot\left(s_{1}-s_{c}\right)
$$

where $\rho \cdot n^{T}$ is constant in both pixels.

Only if the illumination variation is monotonic, it means that $S_{I}$ and $S_{C}$ change in similar proportion and sign, the operator has an invariant behavior. In any other case, more usually presented in real life applications, the LBP changes as the illumination changes, it means that the operator is sensitive to this kind of lighting variations.

\subsection{Discrete Cosine Transform to Compensate for Illumination Variations}

The DCT has been used in some face recognition studies, either in a holistic appearance-based [11] or local appearance-based sense [6]. The general idea is to extract the DCT coefficients and once they are obtained, the top-left one is removed since it only corresponds to the average value of pixels intensities. Of the remaining coefficients the ones containing the highest information are extracted via zig-zag scan to form the feature vector which is used by the classifier. However how many and which coefficients should be extracted to compose the feature vector remains a question.

In [9] is presented a different way of using DCT to compensate for illumination variations. They show that illumination variations can be well compensated by adding or subtracting a compensation term to a given image, in the logarithm domain. This could be easy if we know where illumination variations and important facial features are, but in face images, especially when they are affected with large illumination variations, feature detection is a nontrivial task. 
In a face image, illumination usually changes slowly except for some shadows and specularities on the face. Consequently, illumination variations mainly lie in the lowfrequency band and illumination variations can be reduced by removing lowfrequency components. Then the DCT is used to transform the image from spatial domain to frequency domain. Since illumination variations are expected to be in the low-frequency components, in [9] they proposed setting the low - frequency DCT coefficients to zero in the logarithm domain to compensate for illumination variations, and they obtained promising results. Notice that these low - frequency DCT coefficients are the ones used in the traditional DCT method. They compared their method with a large number of methods [9] and very good results were obtained in illumination problems.

\section{Proposed Method}

After we have analyzed the use of LBP and DCT methods in face recognition, we can say that the LBP operator is sensitive to large variations in illumination conditions and the DCT method in the logarithm domain faces the illumination problems better than LBP does. We then propose a new method which combines some ideas presented in [8] and [9].

In [8] an approach for using LBP method as a preprocessing step and DCT coefficients to form the feature vectors was proposed, and this method shows the best performance in a recent comparison between the most representative extensions and the uses of the original LBP operator [7]. On the other hand, in [9] were presented very good results using DCT of the whole image in the logarithm domain to compensate for illumination variations.

Considering that LBP can represent well the facial structures when the variation in lights are monotonic, that the DCT presents good results facing the illumination problems in the logarithm domain, and that illumination variations affect less in localities than in the whole image, we think that discarding low-frequency DCT coefficients in the logarithm domain in a local way as preprocessing step, and applying LBP method to represent the facial features as such, the robustness against illumination variations can be increased. In Fig. 2 the proposed method is exposed.

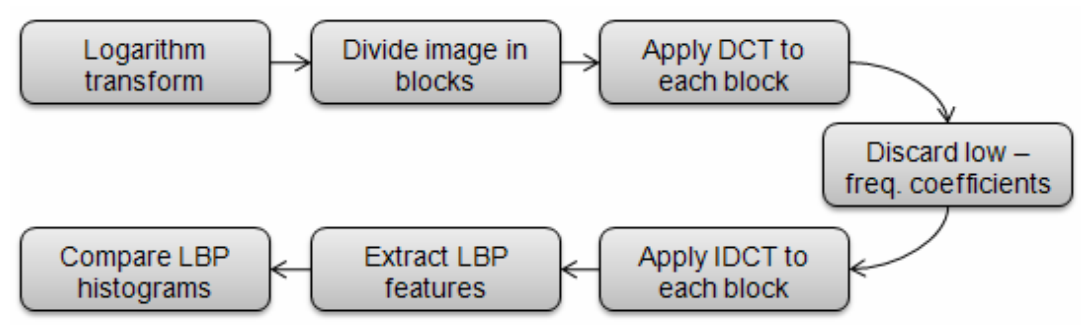

Fig. 2. Overview of the proposed method 


\section{Experiments and Results}

We used the Yale B Face Database [12] to evaluate the proposed method. This face database contains images of 10 subjects each seen under 576 viewing conditions ( 9 poses x 64 illumination conditions). Since we are concerned with the illumination problem in this paper, only the frontal face images were used ${ }^{1}$. To obtain the 64 illumination conditions, the angle between the light source direction and the camera axis was changed every time. The larger the angle, the more unfavourable the lighting conditions are. Then, the frontal face images were divided into five subsets according to this angle, as can be seen in Table 1:

Table 1. Yale B Face Database division according to the lighting variations

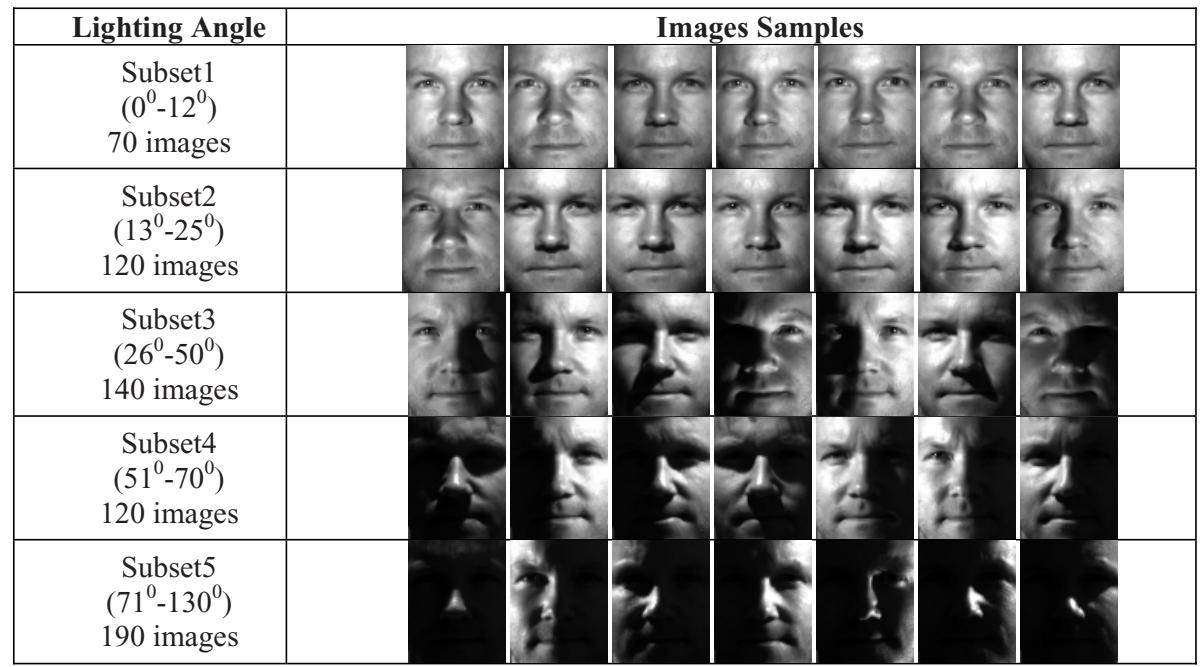

In our experiments, all face images were closely cropped to include only the face region and the extracted face images were geometric normalized by the centres of the two eyes to be $64 \times 80$ pixels in size. The local blocks in which the images were divided, has $8 \times 8$ pixels of size.

The Subset 1 was used as gallery set and each one of the rest subsets were tested using 5 different approaches. First, the original LBP and DCT methods were executed, then the method that used LBP for preprocessing and DCT for classification ${ }^{2}$ (INORM LBP + DCT) and the one using DCT in the logarithm domain with the whole image to compensate for illumination variations (LogDCT_Global + LBP), in this case LBP method was used as feature descriptor rather than the pixel intensities directly as in [9]. Finally our proposal (LogDCT_Local + LBP) was implemented and compared with all of them. The obtained results are shown in Table 2 and Table 3:

\footnotetext{
${ }^{1}$ In the experiments were included the corrupted images of the database.

${ }^{2}$ Best LBP method presented in [7].
} 
Table 2. Equal Error Rate (EER) of the different methods in each subset

\begin{tabular}{|c|c|c|c|c|}
\hline & Subset2 & Subset3 & Subset4 & Subset5 \\
\hline LBP & $5.95 \%$ & $23.46 \%$ & $33.28 \%$ & $37.16 \%$ \\
\hline Local DCT & $3.88 \%$ & $16.62 \%$ & $24.52 \%$ & $22.48 \%$ \\
\hline INORM LBP + DCT & $3.34 \%$ & $12.71 \%$ & $16.42 \%$ & $19.58 \%$ \\
\hline LogDCT_Global + LBP & $3.57 \%$ & $12.44 \%$ & $17.61 \%$ & $18.57 \%$ \\
\hline LogDCT_Local + LBP (Proposal) & $3.15 \%$ & $10.12 \%$ & $15.33 \%$ & $17.29 \%$ \\
\hline
\end{tabular}

Table 3. Recognition rates of the different methods at the first three positions in each subset ${ }^{3}$

\begin{tabular}{|c|c|c|c|c|c|}
\hline & LBP & DCT & $\begin{array}{c}\text { INORM } \\
\text { LBP + DCT }\end{array}$ & $\begin{array}{c}\operatorname{LogDCT}_{-} \\
\text {Global+LBP }\end{array}$ & $\begin{array}{c}\text { LogDCT } \\
\text { Local + LBP }\end{array}$ \\
\hline \multicolumn{6}{|l|}{ Subset3 } \\
\hline Rank 1 & 97.14 & 99.29 & 98.57 & 95.71 & 99.29 \\
\hline Rank 2 & 97.86 & 99.29 & 99.29 & 97.87 & 100 \\
\hline Rank 3 & 97.86 & 99.29 & 99.29 & 98.57 & 100 \\
\hline \multicolumn{6}{|l|}{ Subset4 } \\
\hline \begin{tabular}{|l|} 
Rank 1 \\
\end{tabular} & 65.83 & 88.33 & 90.83 & 89.17 & 93.33 \\
\hline Rank 2 & 75.83 & 91.67 & 92.5 & 90.83 & 96.67 \\
\hline Rank 3 & 79.17 & 93.33 & 95 & 93.33 & 97.5 \\
\hline \multicolumn{6}{|l|}{ Subset5 } \\
\hline Rank 1 & 22.10 & 83.68 & 87.89 & 78.94 & 90.84 \\
\hline Rank 2 & 33.68 & 87.37 & 90.00 & 82.63 & 94.77 \\
\hline Rank 3 & 34.21 & 90.00 & 91.58 & 85.79 & 96.32 \\
\hline
\end{tabular}

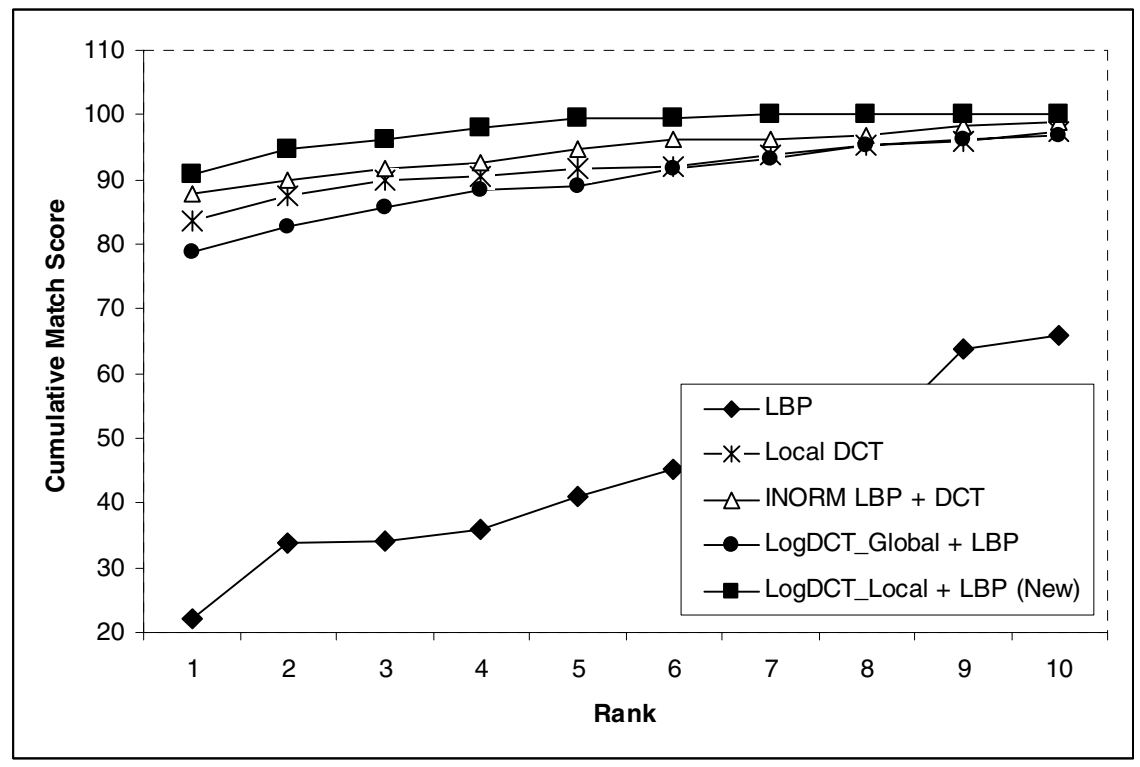

Fig. 3. Cumulative match score vs. rank curve for the proposed method compared with the others in the Subset 5

\footnotetext{
${ }^{3}$ The Subset 2 results were not included since the recognition rate was $100 \%$ in all cases.
} 
In Table 2 it can be seen that the proposed method was the one with lower EER in all subsets, being the less affected by the variations in the light incident angle. From the results obtained with the LBP method, it can be inferred that illumination has a monotonic behavior only when the light incident angle varies in a little magnitude.

Table 3 shows that the proposed method obtains higher recognition rates than the rest of the approaches, from the first positions of the candidates list, in all subsets. Even in Subset5, which has the worst lighting conditions, the recognition rate reaches more than the $90 \%$ from the first position. This can be verified in Fig. 3 in which the cumulative match score vs. rank curve was used to illustrate the performance of the different methods tested in Subset5 (the most difficult subset). In the graphic it can be noticed that the proposed method achieves $100 \%$ of recognition rate in position $7^{\text {th }}$ and none of the rest methods gets this value before the $10^{\text {th }}$ position. The figure also shows the low performance of the LBP method when the illumination variations are large.

\section{Conclusions}

In this work the sensitiveness of the LBP and DCT methods to illumination variations was analyzed. The experimental results confirmed that LBP method works well when the lighting variations correspond to little changes in the light incident angle, but is very sensitive to large variations in illumination conditions. Also, it can be seen that all DCT approaches outperform the LBP method facing the illumination problems. The proposed method, using local DCT in the logarithm domain as preprocessing and LBP to represent the facial structures, outperforms the results of the other approaches in which these methods were used.

\section{References}

1. Li, S.Z., Jain, A.K.: Handbook of Face Recognition. Springer, New York (2005)

2. Phillips, P.J., Scruggs, W.T., O'Toole, A.J., Flynn, P.J., Bowyer, K.W., Schott, C.L., Sharpe, M.: FRVT 2006 and ICE 2006 Large-Scale Results. National Institute of Standards and Technology, Gaithersburg, MD 20899 (2007)

3. Tan, X.Y., Chen, S.C., Zhou, Z.H., Zhang, F.: Face recognition from a single image per person: A survey. Pattern Recognition 39(9), 1725-1745 (2006)

4. Villegas, M., Paredes, R.: Comparison of Illumination Normalization Methods for Face Recognition. In: Third COST 275 Workshop Biometric on the Internet, pp. 27-30 (2005)

5. Ahonen, T., Hadid, A., Pietikäinen, M.: Face recognition with local binary patterns. In: Pajdla, T., Matas, J(G.) (eds.) ECCV 2004. LNCS, vol. 3021, pp. 469-481. Springer, Heidelberg (2004)

6. Ekenel, H.K., Stiefelhagen, R.: Local Appearance Based Face Recognition using Discrete Cosine Transform. In: 13th European Signal Processing Conference (EUSIPCO), Turkey (2005)

7. Marcel, S., Rodriguez, Y., Heusch, G.: On the Recent Use of Local Binary Patterns for Face Authentication. International Journal on Image and Video Processing Special Issue on Facial Image Processing (2007) 
8. Heusch, G., Rodriguez, Y., Marcel, S.: Local Binary Patterns as an Image Preprocessing for Face Authentication. In: IEEE International Conference on Automatic Face and Gesture Recognition (2006)

9. Chen, W., Er, M.J., Wu, S.: Illumination Compensation and Normalization for Robust Face Recognition Using Discrete Cosine Transform in Logarithm Domain. IEEE Transactions on Systems, Man and Cybernetics- Part B 36(2), 458-466 (2006)

10. Marcel, S., Keomany, J., Rodriguez, Y.: Robust-to-illumination face localisation using Active Shape Models and Local Binary Patterns. IDIAP Research Report 47 (2006)

11. Hafed, Z.M., Levine, M.D.: Face Recognition Using the Discrete Cosine Transform. International Journal of Computer Vision 43(3), 167-188 (2001)

12. Georghiades, A.S., Belhumeur, P.N., Kriegman, D.J.: From few to many: illumination cone models for face recognition under variable lighting and pose. IEEE Transactions on Pattern Analysis and Machine Intelligence 23(6), 643-660 (2001) 